\title{
Producción y rentabilidad del frijol como monocultivo y asociado con limón Persa en el norte de
}

\section{Veracruz, México}

Production and profitabity of bean like monoculture and associated with Persian lemon in the north of Veracruz, Mexico

José Raúl Rodríguez Rodríguez ${ }^{1}$, Oscar Hugo Tosquy Valle ${ }^{2}$, Marcos Ventura Vázquez Hernández², Rigoberto Zetina Lezama $^{2 \bowtie}$ y Ulises Díaz Zorrilla ${ }^{1}$

${ }^{1}$ Campo Experimental Ixtacuaco. INIFAP. Km 4.5 Carr. Martínez de la Torre-Tlapacoyan. C.P. 93600. ${ }^{2}$ Campo Experimental Cotaxtla. INIFAP. Km 22.5 Carr. Veracruz-Córdoba, Ver. C.P. 94277

${ }^{凶}$ Autor para correspondencia: zetina.rigoberto@inifap.gob.mx

\section{RESUMEN}

En Martínez de la Torre, Ver., México, existen 16,115 ha establecidas con cítricos que aún no están en producción y pueden aprovecharse para sembrar frijol como cultivo asociado. El objetivo del trabajo fue comparar el rendimiento y rentabilidad del cultivo de frijol sólo y asociado con limón Persa, así como determinar, que sistema de cultivo, es más eficiente en el uso de la tierra. El experimento se estableció en noviembre de 2017 en tres localidades del norte de Veracruz. En cada sistema de cultivo, el frijol se sembró en franjas y ocupó una superficie de $216 \mathrm{~m}^{2}$. Se delimitaron cuatro áreas de siembra de frijol de $6 \mathrm{~m}^{2}$ cada una, por sistema de cultivo, en las que se cuantificaron diferentes variables agronómicas, incluido el rendimiento de grano, las cuales se analizaron en diseño experimental completamente al azar con cuatro repeticiones, considerando los valores de las variables obtenidos en cada área delimitada como una repetición. Para la separación de promedios se aplicó la DMS al 5\%. También se realizó un análisis de relación beneficio-costo y se obtuvo el índice equivalente de la tierra (IET). Con el sistema de cultivo de frijol asociado con limón Persa, se obtuvo un rendimiento promedio de $1430.7 \mathrm{~kg} \mathrm{ha}^{-1}$, significativamente superior al que se produjo en monocultivo. También se obtuvo una utilidad neta por hectárea, $52.3 \%$ superior y la mejor relación beneficio-costo, además de una mayor eficiencia en el uso del terreno (IET de 1.23), que cuando el frijol se estableció en monocultivo.

Palabras clave: Phaseolus vulgaris, Citrus latifolia, asociación de cultivos, eficiencia de uso de la tierra.

\begin{abstract}
In Martinez de la Torre, Veracruz, Mexico, there are 15,115 ha established with citrus trees, which are not yet in production can be used to plant beans as an associated crop. The aim of the present research was to compare the yield and profitability of the bean crop alone or associated with Persian lemon, as well as to determine which bean cultivation system is more efficient in the use of the land. The experiment was established in November 2017 in three localities in northern Veracruz. In each cultivation system, beans were planted in strips and occupied a surface of $216 \mathrm{~m}^{2}$. Four bean planting


areas of $6 \mathrm{~m} 2$ each were delimited, by crop system, in which different agronomic variables were quantified, including grain yield, which were analyzed in a completely randomized experimental design with four replications, considering the values of the variables obtained in each area delimited as a repetition. The 5\% DMS test was use for separation of averages. A benefit-cost ratio analysis was also carried out and the land equivalence ratio (LER) was obtained. With the bean cultivation system associated with Persian lemon, an average yield of $1430.7 \mathrm{~kg} \mathrm{ha}^{-1}$ was obtained, significantly higher than that produced in monoculture. There was also a net profit per hectare, $52.3 \%$ higher and the best benefit-cost ratio, in addition to a greater efficiency in land use ratio (LER of 1.23), than when the beans were established in monoculture.

Key words: Phaseolus vulgaris, Citrus latifolia, intercropping, land equivalence ratio.

\section{INTRODUCCIÓN}

El frijol común (Phaseolus vulgaris L.) es la leguminosa más importante en el mundo, la principal fuente de proteína vegetal y la más barata. En México el consumo per cápita es de alrededor de $9 \mathrm{~kg}$ por persona por año, con una tendencia a la baja; es el tercer cultivo en importancia por su superficie sembrada, sólo después del maíz y sorgo de grano. En 2014 se cosecharon 1'680,000 ha en el país; en ese mismo año en la región de Martínez de la Torre, Veracruz se sembraron 1,808 ha de frijol, y para 2017 sólo sembraron 1,512 ha, lo que significa una reducción del $16.37 \%$. En Martínez de la Torre existe una superficie establecida con cítricos de 230,218 ha, de las cuales poco más del $7 \% \quad(16,115$ ha $)$ corresponde a plantaciones nuevas (SIAP, 2017), que aún no están en producción y pueden aprovecharse para sembrar frijol entre las hileras. La asociación de cultivos consiste en plantar dos o más especies al mismo tiempo y en el mismo terreno (FAO, 2017a). El frijol es una de las especies que más se utiliza en la asociación principalmente con maíz (Kohashi, 1990) u otros cultivos como calabaza y caña de azúcar (Balderas y González, 2013); en árboles como el cocotero, los cítricos y el aguacate se puede sembrar el área intermedia con cultivos como el café, pero especialmente con cultivos alimentarios como leguminosas. El sistema de producción frijol asociado con cítricos puede ser una buena alternativa en la región, ya que se logra un aprovechamiento óptimo del suelo, tanto en su ocupación, como en el consumo de nutrientes. Al cubrir el terreno, se evita el crecimiento de las malezas, y se mantiene el suelo limpio, debido a las escardas que se realizan en el frijol; también se evita la erosión del suelo por efecto del agua y del viento, además, se conserva mayor humedad, ya que se evita la evaporación; esta asociación también proporciona protección contra daños por enfermedades y plagas, ya que modifica el microclima (Gómez y Zavaleta, 2001; Lithourgidis et al., 2011). El frijol como leguminosa tiene la capacidad de fijar nitrógeno atmosférico, favoreciendo la fertilidad del terreno (Paredes, 2013; FAO, 2017b); no existe competencia interespecífica por luz, ya que el crecimiento del frijol es menor al de los cítricos, por lo que no hay interferencia en la cantidad de energía luminosa y se puede realizar a plenitud la fotosíntesis, pues se aprovecha toda la radiación fotosintéticamente activa (Kohashi, 1990). Con base en lo anterior, los sistemas de cultivos asociados presentan mayor eficiencia productiva en comparación con los de monocultivo (Molina-Anzures et al., 
2016). El objetivo del trabajo de investigación fue comparar el rendimiento y rentabilidad de este cultivo sólo y asociado con cítricos (específicamente limón Persa), así como determinar, que sistema de cultivo de frijol, es más eficiente en el uso de la tierra.

\section{MATERIALES Y MÉTODOS}

El experimento se estableció en noviembre de 2017, en tres localidades de la zona citrícola de Martínez de la Torre, en el norte del estado de Veracruz: Campo Experimental Ixtacuaco, ubicado en el municipio de Tlapacoyan, $\left(20^{\circ}\right.$ $02^{\prime} \mathrm{LN}, 97^{\circ} 5^{\prime}$ LO y $88 \mathrm{~m}$ de altitud); María de la Torre, municipio de Martínez de la Torre, (20 12' LN, 96 99' LO, y 40 m de altitud), y El Cortijo, municipio de Tlapacoyan $\left(20^{\circ} 02^{\prime}\right.$ LN, 97 $14^{\circ}$ ' LO y $120 \mathrm{~m}$ de altitud). Se evaluaron dos tratamientos: frijol asociado con limón Persa (Citrus latifolia) y frijol en monocultivo. En ambos sistemas de cultivo, se sembró el genotipo de frijol T-39 de hábito de crecimiento determinado arbustivo tipo II y grano negro, opaco y pequeño (Voysest, 2000), muy parecido al de la variedad Negro Michigan, de uso común en el norte de Veracruz.

La siembra se realizó en franjas, en surcos separados a $0.60 \mathrm{~m}$ y cada tratamiento ocupó una superficie de $216 \mathrm{~m}^{2}$. Durante la fase vegetativa del cultivo se controló la maleza mediante dos escardas manuales, se fertilizó con la fórmula $40 \mathrm{~N}-40 \mathrm{P}_{2} \mathrm{O}_{5}-00 \mathrm{~K}_{2} \mathrm{O}$, mediante la aplicación de urea (46-00-00) y DAP (18-4600), cuando en las plantas aparecieron las hojas primarias. Por la presencia de doradilla (Diabrótica balteata) y chicharrita (Empoasca kraemeri), se aplicó cipermetrina en dosis de $200 \mathrm{~mL} \mathrm{ha}^{-1}$ de producto comercial.

Durante la conducción de ensayos se registró la precipitación pluvial $(\mathrm{mm})$, la temperatura media $\left({ }^{\circ} \mathrm{C}\right)$ y la humedad relativa del ambiente (\%). En cada sistema de cultivo, se delimitaron cuatro áreas de siembra de frijol seleccionadas al azar de $6 \mathrm{~m}^{2}$ cada una (dos surcos de $5 \mathrm{~m}$ de longitud), en las que se cuantificaron las siguientes variables: 1. Días a floración media, contabilizados a partir de la siembra hasta que en el $50 \%$ de las plantas tenían por lo menos una flor abierta; 2. Días a madurez fisiológica, a partir de la siembra hasta que en el $50 \%$ de las vainas cambiaron de color verde a amarillo; 3 . Reacción del frijol, solo y asociado, ante la presencia del hongo que provoca la enfermedad del moho blanco, la cual incidió en la localidad del Campo Experimental Ixtacuaco. Esta evaluación se realizó durante la etapa R8 de llenado de vainas (Fernández et al., 1985), utilizando la escala de 1 a 9 para la evaluación de germoplasma del CIAT (1987), donde 1 - 3 = Resistente; 4 - 6 = Reacción intermedia y 7 9 = Susceptible. 4. Número de plantas cosechadas, en cada área delimitada; 5. Número de vainas por planta, determinado en 10 plantas con competencia completa, tomadas al azar en cada área delimitada, en la etapa de cosecha, a las cuales se les contabilizaron sus vainas y se obtuvo el promedio; 6. Número de granos por vaina, obtenido de 20 vainas tomadas al azar en cada área delimitada, a las que se les determinó la cantidad de semilla por vaina y se obtuvo su promedio; 7. Peso de 100 granos, determinado en cuatro muestras de 100 granos tomados al azar en cada área delimitada, de las cuales se obtuvo el promedio en gramos. 8. Rendimiento de grano, que se calculó a partir del peso del grano cosechado y limpio de cada área delimitada, en kilogramos por hectárea al $14 \%$ de humedad.

Los datos de las variables cuantificadas en ambos sistemas de cultivo de frijol, se analizaron con el paquete estadístico de la Universidad Autónoma de Nuevo León versión 
2.5 (Olivares, 1994), mediante el diseño experimental completamente al azar con cuatro repeticiones, considerando los valores de las variables obtenidos en cada área delimitada como una repetición. Para la separación de promedios se aplicó la prueba basada en la Diferencia Mínima Significativa al 5\% de probabilidad del error. También se realizó un análisis económico tomando como base los costos fijos + los costos variables, para obtener el costo total, de tal manera que la utilidad neta fue igual al rendimiento $\left(\mathrm{kg} \mathrm{ha}^{-1}\right)$ por el precio de $1 \mathrm{~kg}$ de frijol menos el costo total. Para este análisis se consideró un costo de producción de frijol por hectárea en ambos sistemas de cultivo de $\$ 11,537.90$ y un precio de venta de $\$ 18.00$ por kilogramo de frijol.

Para calcular el Índice equivalente de la Tierra (IET) se utilizó el rendimiento relativo del frijol asociado (RA) dividido entre el rendimiento del frijol en monocultivo (RM) (Willey y Rao., 1980; Mead y Willey, 1980). Si el valor que se obtiene es $>1$ la asociación es ventajosa; si el valor obtenido es $=1$ es indiferente y si el resultado es $<1$, el monocultivo es mejor. Sólo se consideró el rendimiento del frijol, debido a que el limón Persa aún no se encontraba en producción.

\section{RESULTADOS Y DISCUSIÓN}

En las localidades de evaluación, las condiciones de precipitación pluvial (324 a $438.3 \mathrm{~mm}$ ), temperatura media (19.37 a 21.6 $\left.{ }^{\mathrm{O}} \mathrm{C}\right)$ y humedad relativa (79.02 a $\left.85.62 \%\right)$ durante el ciclo del cultivo, fueron adecuadas para el buen desarrollo del cultivo de frijol (CEIXTA, 2018; WWO, 2018), ya que este cultivo requiere de 300 a $500 \mathrm{~mm}$ de precipitación pluvial bien distribuidos durante el ciclo, con una temperatura óptima de entre 18 y $24^{\circ} \mathrm{C}$ (Ruiz-Corral et al., 2013) y humedad relativa de moderadamente seca a húmeda (79 a $85 \%)$.

En los análisis de varianza combinados de los tres sitios de prueba, sólo se detectaron diferencias significativas $(p \leq 0.05)$ entre tratamientos en las variables peso de 100 granos y rendimiento de grano.

En el Cuadro 1 se muestra que, en ambos sistemas de cultivo, la floración y la madurez fisiológica del frijol ocurrieron en un tiempo estadísticamente similar, lo cual coincide con lo reportado por Singh et al. (1991), quienes también encontraron muy poca variación en el número de días en que el frijol llegara a la etapa de floración (Cuadro 1). Cabe resaltar que el genotipo T39 mostró uniformidad en su ciclo de madurez y buena adaptación en los sitios de prueba.

Con respecto a la incidencia del moho blanco en la localidad de Campo Experimental Ixtacuaco, en el análisis de varianza individual se detectó significancia estadística $(p \leq 0.05)$ entre sistemas de cultivo. El frijol sembrado como monocultivo mostró una incidencia de moho blanco de 5, significativamente superior a la que se presentó en el frijol asociado con limón Persa (calificación de 2) (datos no mostrados). Los menores daños presentados en este último sistema de cultivo, se deben en gran parte, a que hay una modificación del microclima, disminución de la temperatura del aire y menor evaporación, lo que provoca mayor frescura en el cultivo de frijol asociado por efecto del sombreado (Gómez y Zavaleta., 2001; Lithourgidis 2011).

En el Cuadro 1 también se muestra que, aunque en los dos sistemas de cultivo, se cosechó una cantidad de plantas de frijol estadísticamente similar, numéricamente en el frijol asociado con limón Persa se tuvo una cantidad de plantas considerablemente mayor, lo cual se traduce en una mayor cantidad de vainas y granos 
producidos por hectárea, lo que consecuentemente incrementa el rendimiento de frijol (Westermann y Crothers, 1977; White, 1991; Rodríguez et al., 2011), pese a que el número promedio de vainas producidas por planta y de granos por vaina hayan sido similares estadísticamente en ambos sistemas de cultivo (Gonçalves et al., 2003). A su vez, aunque se registró un peso de grano mayor en el cultivo de frijol asociado, éste fue estadísticamente similar al frijol establecido en monocultivo (Cuadro 1). Se tiene conocimiento de que esta característica es controlada por un gran número de factores genéticos y afectada por el sitio y el ambiente de cada sistema (Ribeiro et al., 2003; Rodríguez et al., 2011).

Cuadro 1. Comportamiento agronómico promedio de tres localidades del genotipo de frijol T39 sólo y asociado con limón Persa. Ciclo de otoño-invierno de 2017-18.

\begin{tabular}{lcccccc}
\hline \multicolumn{1}{c}{ Sistema de cultivo } & $\mathrm{F}(\mathrm{d})$ & $\mathrm{MF}(\mathrm{d})$ & $\mathrm{PC}$ & $\mathrm{VP}$ & $\mathrm{GV}$ & $\mathrm{PG}$ \\
\hline Frijol - limón Persa & $42.7 \mathrm{a}$ & $84.9 \mathrm{a}$ & $73.7 \mathrm{a}$ & $19.9 \mathrm{a}$ & $6.1 \mathrm{a}$ & $20.9 \mathrm{a}$ \\
Frijol en monocultivo & $42.5 \mathrm{a}$ & $85.5 \mathrm{a}$ & $64.0 \mathrm{a}$ & $19.1 \mathrm{a}$ & $6.1 \mathrm{a}$ & $20.2 \mathrm{a}$ \\
Promedio & 42.65 & 85.20 & 68.85 & 19.50 & 6.10 & 20.55 \\
ANVA & $\mathrm{ns}$ & $\mathrm{Ns}$ & $\mathrm{ns}$ & $\mathrm{ns}$ & $\mathrm{ns}$ & $\mathrm{ns}$ \\
$\mathrm{CV}(\%)$ & 2.329 & 0.770 & 15.444 & 20.611 & 5.984 & 4.240 \\
\hline
\end{tabular}

$\mathrm{F}=$ Floración; $\mathrm{MF}=$ Madurez fisiológica; $\mathrm{PC}=$ Plantas cosechadas; $\mathrm{VP}=$ Vainas por planta; $\mathrm{GV}=$ Granos por vaina; $\mathrm{PG}=$ Peso de 100 granos.

Con el sistema de cultivo de frijol asociado con limón Persa, se obtuvo un rendimiento promedio significativamente superior al que se produjo en monocultivo, lo cual se atribuye en gran parte, a una mayor sanidad del cultivo, debido a que las plantas de frijol disponen de un mejor ambiente de humedad y temperatura para un adecuado desarrollo y una mayor eficiencia en la utilización de los elementos disponibles (Lithourgidis et al., 2011) (Cuadro 2).

Cuadro 2. Rendimiento de grano de frijol $\left(\mathrm{kg} \mathrm{ha}^{-1}\right)$ en dos sistemas de cultivo y tres sitios de prueba, en el norte de Veracruz. Ciclo de otoño-invierno de 2017-18.

\begin{tabular}{lcccc}
\hline \multicolumn{1}{c}{ Sistema de cultivo } & CEIXTA & $\begin{array}{c}\text { María } \\
\text { de la Torre }\end{array}$ & $\begin{array}{c}\text { El Cortijo } \\
\text { Rendimiento } \\
\text { de grano }\end{array}$ \\
\hline Frijol asociado con cítricos & 1734.70 & 1303.32 & 1254.00 & $1430.67 \mathrm{a}$ \\
Frijol en monocultivo & 1324.07 & 1234.48 & 919.75 & $1159.41 \mathrm{~b}$ \\
Promedio & 1529.38 & 1268.90 & 1086.87 & 1295.04 \\
DMS $(0.05)$ & & & & 184.708 \\
CV $(\%)$ & & & & 15.44 \\
\hline
\end{tabular}

Letras diferentes en la misma columna son estadísticamente diferentes de acuerdo a la DMS, 0.05.

De acuerdo al análisis económico, el mayor beneficio neto se obtuvo cuando el frijol se asoció con limón Persa, con el cual se obtuvo una relación beneficio-costo de 1:2.23, que indica que con este sistema de cultivo, el productor por cada peso que invierte, va a 
recuperar su inversión y tener una ganancia de $\$ 1.23$, en tanto que, con el sistema de frijol en monocultivo la relación beneficio-costo fue de $1: 1.81$, es decir, que con la siembra de frijol asociado se obtuvo una ganancia neta $52.3 \%$ superior al sistema tradicional de siembra de frijol en monocultivo (Cuadro 3).

Cuadro 3. Beneficio neto del frijol establecido en dos sistemas de cultivo en tres localidades del norte de Veracruz. Ciclo de otoño-invierno de 2017-18.

\begin{tabular}{ccccc}
\hline Sistema de cultivo & $\begin{array}{c}\text { Rendimiento } \\
\left(\mathrm{kg} \mathrm{ha}^{-1}\right)\end{array}$ & $\begin{array}{c}\text { Costo de producción } \\
\left(\$ \mathrm{ha}^{-1}\right)\end{array}$ & $\begin{array}{c}\text { Beneficio bruto } \\
\left(\$ \mathrm{ha}^{-1}\right)\end{array}$ & $\begin{array}{c}\text { Benefício neto } \\
\left(\$ \mathrm{ha}^{-1}\right)\end{array}$ \\
\hline Frijol - limón Persa & $1,430.67$ & $11,537.90$ & $25,752.06$ & $14,214.16$ \\
Frijol en monocultivo & $1,159.41$ & $11,537.90$ & $20,869.38$ & $9,331.48$ \\
\hline
\end{tabular}

Según el Índice Equivalente de la Tierra (IET), se necesitan 1.23 ha de frijol en monocultivo para obtener la misma producción que cuando este cultivo se encuentra asociado con limón Persa. De acuerdo a los resultados obtenidos en este estudio, este último sistema representa una buena opción para los productores citrícolas del norte de Veracruz, pues permite hacer un uso más eficiente de la tierra en cuanto a productividad y rentabilidad de la unidad de producción (Mead y Willey 1980; MolinaAnzures et al., 2016).

Cuadro 4. Índice de Equivalencia de la Tierra (IET) obtenido en dos sistemas de cultivo y tres localidades de evaluación del norte de Veracruz. Ciclo de otoño-invierno de 2017-18.

\begin{tabular}{cccc}
\hline Sistema de cultivo & $\begin{array}{c}\text { Rendimiento } \\
\left(\mathrm{kg} \mathrm{ha}^{-1}\right)\end{array}$ & $\mathrm{RA}^{\mathrm{R}}$ & $\mathrm{IET}^{\dagger}$ \\
\hline Frijol asociado & $1,430.67$ & $1430.67 / 1159.41$ & 1.23
\end{tabular}

Frijol en monocultivo

$1,159.41$

$\mathrm{RA} / \mathrm{RM}^{\dagger \dagger}=$ Rendimiento asociado/Rendimiento en monocultivo; IET $\dagger=$ Índice de Equivalencia de la Tierra.

\section{CONCLUSIONES}

En las tres localidades de evaluación, el rendimiento de frijol fue significativamente mayor cuando este cultivo se estableció asociado con limón Persa, por lo que este sistema representa una buena alternativa de producción para los productores citrícolas de la región de Martínez de la Torre, en el norte del estado de Veracruz.
Con este mismo sistema de cultivo de frijol, se obtuvo una utilidad neta por hectárea $52.3 \%$ superior y la mejor relación beneficio-costo, además de una mayor eficiencia en el uso del terreno (IET de 1.23), que con el sistema de cultivo de frijol en monocultivo. 


\section{LITERATURA CITADA}

Balderas, F., e I. González. 2013. Cocotero híbrido intercalado con cultivos anuales y perennes, tecnología sustentable. Revista Mexicana de Ciencias Forestales. 4 (20): 58-71.

CEIXTA (Campo Experimental Ixtacuaco), 2018. Estación climatológica. Datos históricos de precipitación $(\mathrm{mm})$, temperatura $\left({ }^{\mathrm{O}} \mathrm{C}\right)$ y humedad relativa $(\%)$, 2017-2018. www.clima.inifap.gob.mx (consultado el 18 abril de 2018).

CIAT (Centro Internacional de Agricultura Tropical). 1987. Sistema estándar para la evaluación de germoplasma de frijol. Schoonhoven, A. van y M. A. PastorCorrales (comps.). Cali, Colombia. 56 p.

CIAT (Centro Internacional de Agricultura Tropical). 1988. El moho blanco y su manejo. Guía de estudio. Contenido científico. H. F. Schwartz; M. PastorCorrales. Cali, Colombia. 38 p.

FAO. 2017a). Mejorando la nutrición a través de huertos y granjas familiares. Cultivos asociados. Cartilla Tecnológica No. 10. www.fao.org (consultado 23 mar 2017).

FAO. 2017b). Información básica. Mejoramiento del suelo. Cartilla Tecnológica. No. 5. www.fao.org (consultado 23 mar 2017).

Fernández, F., P. Gepts y M. López. 1985. Etapas de desarrollo en la planta de frijol. p. 61-78. In: López, M., F. Fernández y A. van Schoonhoven (eds). Frijol: investigación y Producción. Programa de la ONU para el Desarrollo (PNUD). Centro Internacional de Agricultura Tropical (CIAT). Cali, Colombia.

Gómez Rodríguez Olga y Emma Zavaleta Mejía. 2001. La asociación de cultivos una estrategia más para manejo de enfermedades, en particular con Tagetes spp. Rev. Mex. de Fitopatología. 19 (1) 94-99.

Gonçalves, M., Correa, A., Destro, D., Ferreira, L. y Alves, T. 2003. Correlations and path analysis of common bean grain yield and its primary components. Crop Breeding and Applied Biotech. 3 (3): 217-222. https://doi.org/10.12702/1984-7033.v03n0 $3 \mathrm{a} 06$

Kohashi-Shibata, J. 1990. Aspectos de la morfología y fisiología del frijol (Phaseolus vulgaris L.) y su relación con el rendimiento. Centro de botánica. Colegio de Postgraduados. Chapingo, Méx. 44 p.

Lithourgidis, A., C. Dordas, C. Damalas, D. Vlachostergios. 2011. Annual intercrops: an alternative pathway for sustainable agricultura. Australian Journal Crop Sci. 5(4):396-410.

Mead, R. and Willey, R.W. 1980. The concept of Land Equivalent Ratio (LER) and advantages in yields from intercropping. Experimental Agriculture. 16, 217-228. https://doi.org/10.1017/S00144797000109 78

Molina-Anzures M, J. L. Chavez-Servia, A. Gil-Munoz, P. A. López, E. HernándezRomero, E. Ortiz-Torres. 2016. Eficiencias productivas de asociaciones de maiz, frijol y calabaza (Curcurbita pepo L.), intercaladas con árboles frutales. International Journal of Experimental Botany. 85: 36-50. https://doi.org/10.32604/phyton.2016.85.0 36

Olivares, S. E. 1994. Paquete estadístico de diseños experimentales (programa de cómputo) versión 2.5. Facultad de Agronomía de la Universidad Autónoma de Nuevo León. Marín, N. L., México. 
Paredes, M. C. 2013. Fijación biológica de nitrógeno en leguminosas y gramíneas. Facultad de Ciencias Agrarias. Univ. Católica Argentina. www.uca.edu.ar (consultado marzo 2017)

Ribeiro, N.; L. Hoffmann-Júnior; M. Stroschein and S. Possebon. 2003. Genotype x environment interaction in common bean yield and components. Crop Breeding and Applied Biotech. 3 (1):27-34. https://doi.org/10.12702/1984-7033.v03n0 $1 \mathrm{a} 04$

Rodríguez, J. R., O. Tosquy-Valle, y E. LópezSalinas. 2011. Producción de grano de frijol y su relación con la densidad de plantas, en Ver., Méx. p. 37-42. In: Memoria IV Congreso Internacional Biológico-Agropecuario. Tuxpan, Ver.

Ruíz-Corral, J.A., G. Medina, I. González, H. Flores, G. Ramírez, C. Ortiz, K. Byerly, R. Martínez-Parra. 2013. Requerimientos agroecológicos de los cultivos. CIRPAC. Campo Experimental Centro Altos de Jalisco. Tepatitlán de Morelos, Jal. Libro Técnico Núm. 3. 564 p. ISBN: 978-60737-0188-4.

SIAP (Servicio de Información Agroalimentaria y Pesquera). 2016. Cierre de la producción agrícola por cultivo. www.siap. gob.mx (consultado 3 de abril 2017)
Singh, S., P. Gepts and D. Debouck. 1991. Races of common bean (Phaseolus vulgaris, Fabaceae). Economy Botany. 45(3): 379-396. https://doi.org/10.1007/BF02887079

Voysest, O. 2000. Mejoramiento genético del frijol (Phaseolus vulgaris L.): Legado de variedades de frijol de América Latina 1930-1999. CIAT. Cali, Colombia. 195 p.

White, J. 1991. Conceptos básicos en fisiología de frijol. In: Frijol: Investigación y producción. López, M., F. Fernández y A. van Schoonhoven (eds.) CIAT. Cali, Colombia. pp. 43-56.

Westermann D. and S. Crothers. 1977. Plant population effects on the seed yield components of beans. Crop Science.17: 493-496

https://doi.org/10.2135/cropsci1977.00111 83X001700040002x

Willey, R., R. Rao. 1980. A competitive ratio for quantifying competition between intercropping. Exp. Agric. 16:117-125 https://doi.org/10.1017/S00144797000108 02

WWO (World Weather Online). 2018. Martínez de la Torre Historical Weather, México. http//www.worldweatheronline.com (consultado el 18 de abril de 2018). 
Copyright (c) 2018 José Raúl Rodriguez Rodriguez, Oscar Hugo Tosquy Valle, Marcos V entura Vázquez Hernández, Rigoberto Zetina Lezam a y Ulises Diaz Zorrilla

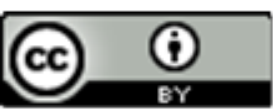

Este tex to está protegido por una licencia CreativeCommons 4.0.

Usted es libre para Compartir —copiar y redistribuir el material en cualquier medio o form ato- y Adaptar el documento - remezclar, transformar y crear a partir del material- para cualquier propósito,, incluso para fines comerciales, siempre que cumpla la condición de:

Atribuci ón: Usted debe dar crédito a la obra original de manera adecuada, proporcionar un enlace a la licencia, e in dicar si se han realizado cam bios. Puede hacerlo en cualquier form a razonable, pero no de form a tal que sugiera que tiene el apoyo del licenciante o 10 recibe por el uso que hace de la obra.

Resumendelicencia - Textocompletodelalicencia 